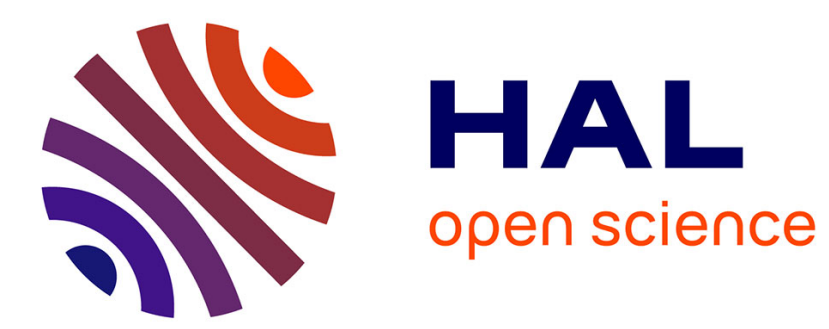

\title{
Complexity of quantifier elimination in the theory of algebraically closed fields
}

Alexander Chistov, Dima Grigoriev

\section{To cite this version:}

Alexander Chistov, Dima Grigoriev. Complexity of quantifier elimination in the theory of algebraically closed fields. Lecture Notes in Computer Science, 1984. hal-03053144

\section{HAL Id: hal-03053144 \\ https://hal.science/hal-03053144}

Submitted on 10 Dec 2020

HAL is a multi-disciplinary open access archive for the deposit and dissemination of scientific research documents, whether they are published or not. The documents may come from teaching and research institutions in France or abroad, or from public or private research centers.
L'archive ouverte pluridisciplinaire HAL, est destinée au dépôt et à la diffusion de documents scientifiques de niveau recherche, publiés ou non, émanant des établissements d'enseignement et de recherche français ou étrangers, des laboratoires publics ou privés. 
COMPLEXITY OF QUANTIFIER ELIMINATION

IN THE THEORY OF ALGEBRAICALLY CLOSED FIELDS

\author{
A.L.Chistov, D.Yu.Grigor'ev \\ Leningrad Scientific Research Computer Centre \\ of the Academy of Sciences of the USSR, \\ Mendeleevskaya 1, Leningrad, 199164, USSR \\ Leningrad Department of V.A.Steklov Mathematical \\ Institute of the Academy of Sciences of the USSR, \\ Fontanka 27, Leningrad, 191011, USSR
}

\begin{abstract}
.
An algorithm is described producing for each formula of the first order theory of algebraically closed fields an equivalent free of quantifiers one. Denote by $N$ a number of polynomials occuring in the formula, by $d$ an upper bound on the degrees of polymomials, by $n$ a number of variables, by a a number of quantifier alternations (in the prefix form). Then the algorithm works within the polynomial in the formula's size and in $(N d)^{n^{(2 a+l)}}$ time. Up to now a bound $(N d)^{n^{\sigma(n)}}$ was known $([5],[7],[15])$.

1. Fast algorithms for factoring multivariable, polymomials and for solving systems of algebraic equations

Lately the considerable progress in the polynomial factoring problem was achieved. Lenstra A.K., Lenstra H.W., Lovasz L. [12] have designed an ingenious polynomial-time algorithm for factoring onevariable polynomials over $\mathbb{Q}$. Independently Kaltofen E. [8] , [9] has constructed a reduction of multivariable factoring over $\mathbb{Q}$ to onevariable factoring, running within the polynomial-time provided that the number of variables is fixed. The authors [1], [4] , have suggested a polynomial-time algorithm for factoring multivariable polynomials over $\mathbb{Q}$ and over finite fields. Later another polymomial-time algorithm for the case of finite fields was exhibited in [13] spreading the method [12].

Also an essential progress has taken place in another important
\end{abstract}


problem of the commutative computeralgebra, namely in the problem of solviag systems of algebraic equations. Earlier a complexity bound of the order $d^{n}$ was known for it,e.g. from [5], [7], [15]. Lazard D. [11] has designed an algorithm for solving homogeneous systems of algebraic equations in the case when the variety of roots in the projective space of the system is null-dimensional, i.e. finite, working within the time $d^{0(n)}$ if the coefficients of the input system are taken from a finite field (certainly, provided that we are supplied with a polynomial-time algorithm for polynomial factoring). The authors [2], [3], [4] involving the polynomial-time algorithr for polynomial factoring [1], [4] and the method from [11] have constructed an algorithm for solving an arbitrary system of algebraic equations, runing within a polynomial in the size $L_{2}$ of the input data (system) and in $d^{n^{2}}$ time. Moreover, the algorithm finds all the irreducible compounds $W_{\alpha} \subset \mathbb{P}^{n}(\bar{F})$ of the variety of roots of the homogeneous system within the polymomial time in $d^{\text {nC }}$ and in $L_{\alpha}$ where $c=1+m a x_{\alpha} \operatorname{dim} W_{\alpha}$ (the general case is reducible here to homogeneous one). Pinding $W_{d}$ allows to answer the principle questions, e.g. emptiness, dimension of the variety of roots. Now we torn ourselves to the exact formulations of the mentioned results. Let a ground fleld $F=H\left(T_{1}, \ldots, T_{\ell}\right) \quad[\eta]$ $H=Q$ or $H=F_{q x}, q=\operatorname{char}(H)$, the elements $T_{1}, \ldots, T_{l}$ be alsebraically independent over $H$; the element $\eta$ is separable

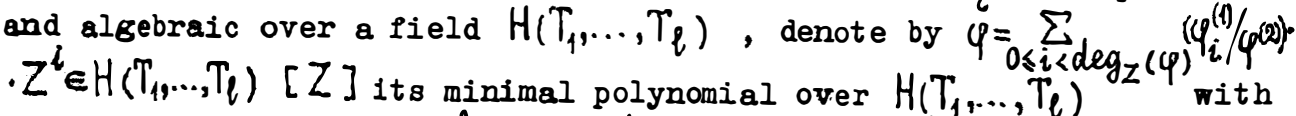
the leading coefficient $l C_{Z}(\varphi)=\left\{\right.$, herewith $\varphi_{i}^{(1)}, \varphi(2) \in H\left[T_{1}, \ldots, T_{l}\right]$ and the degree $\operatorname{deg}\left(\varphi^{(2)}\right)$ is the least possible. Any polynomial $f \in$ $F\left[X_{0} \ldots, X_{n}\right]$ can be uniquely represented in a form $f=\sum$ $\left(a_{i, i_{0}, \ldots, i_{n}} / b\right) \eta^{i} X_{0}^{i_{0}} \ldots X_{n}^{i_{n}}$ where $a_{i, i_{0}} \ldots, i_{n}, b \in H^{\prime}\left[T_{1}, \ldots, T_{l}\right]$, the degree $\operatorname{deg}(b)$ is the least possible; the polynomials $a_{i}, i_{0}, \ldots, i_{n}, b$ are determined uniquely up to a factor from $H^{*}$. Set $d_{2 g} f_{j} f^{\prime}=$ $\max _{i, v_{0} \ldots, i_{n}}\left\{\operatorname{deg}_{T_{j}}\left(a_{i, i_{0}}, \ldots, i_{n}\right), \operatorname{llg}_{T_{j}}(b)\right\}$. By a length of description $l(h)$ in the case $h \in \mathbb{Q}$ we mean its bitwise length, and in the case $h \in \mathbb{F}_{q x}$ we mean $x \log _{2}(q)$. By $l(f)$ denote the maximum of the lengths of descriptions of the coefficients from $H$ in the monomials in $T_{1}, \ldots$, $T_{l}$ of the polymomials $a_{i_{1}, i_{0}, \ldots, i_{n}}, b$. Let $\left.\operatorname{deg}_{x_{j}}(f)<r, \operatorname{deg}_{T_{j}}(f)<r_{2}\right) \operatorname{deg}_{T_{j}}(\varphi)<\psi_{1}, \operatorname{deg}_{z}(\varphi)<\tau_{1}$, $\ell(f) \leqslant M_{2}, l(\varphi) \leqslant M_{1}$. As a size $L_{1}(f)$ of the polynomial $f$ we con-

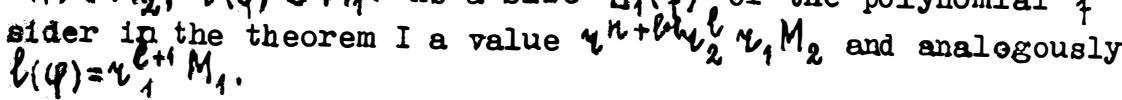


THEOREM I. ( [1], [4] ). One can factor the polynomial $f$ over

$F$ within the polynomial in $L_{1}(f), L_{1}(\varphi), q$ time.

Remark that it is possible within the same time to obtain also

the absolute factorization of $f$ i.e. the factors irreducible

over the algebraic closure $\bar{F}$ of the field $F$ ( [2], [4]).

Proceed to the problem of solving systems of algebraic equations. Let an input system of algebraic equations $f_{0}=\ldots=f_{k}=0$ be given (we can assume w.l.0.g. that $f_{0}, \ldots, f_{k}$ are linearly independent). As a matter of fact we suggest an algorithm which decomposes an arbitrary projective variety on the irreducible compounds, so one can suppose w.1.o.g. that $f_{0}, \ldots, f_{k} \in F\left[X_{0}, \ldots, X_{n}\right]$ are homogeneous relatively to $X_{0}, \ldots, X_{n}$ polynomials. Let $\operatorname{deg}_{T_{1}, \ldots, T_{l}, z}(\varphi)<d_{1}, l\left(f_{i}\right) \leqslant M_{2}$, $\operatorname{deg}_{X_{0}}, \ldots, X_{n}\left(f_{i}\right)<d, \operatorname{deg}_{T_{1}, \ldots, T_{l}}\left(f_{i}\right)<d_{2}$ for all $0 \leqslant i \leqslant k \quad$ and in the theorem 2 a size $L_{2}\left(f_{i}\right)=\left(\begin{array}{c}d+n \\ n\end{array}\right) d_{1} d_{2}^{l} M_{2} \quad$ and $L_{2}(\varphi)=d_{1}^{l+1} M_{1}$. Denote $L=L_{2}\left(f_{0}\right)+\ldots+L_{q}\left(f_{k}\right)$.

The projective variety $\left\{f_{0}=\ldots=f_{k}=0\right\} \subset \mathbb{P}^{k}(\vec{F})$ of roots of the system $f_{0}=\ldots=f_{k}=0$ is decomposable on the compounds $\left\{f_{0}=\right.$ $\left.=f_{k}=0\right\}=\bigcup_{d} W_{\alpha}, \quad$ herewith each compound $W_{d}$ is defined and irreducible over the maximal purely inseparable extension $F^{q^{-\infty}}$ of $F$. Moreover $W_{\alpha}=W_{\alpha \beta}$ where the (absolutely irreducible) compounds $W_{\alpha \beta}$ are defined and irreducible over $\bar{F}$. Denote $c=$ $1+\max \operatorname{dim} W_{\alpha}$. The algorithm designed in [2], [3], [4] finds all $W_{\alpha}$ and thereupon $W_{\alpha \beta}$ (actually, $W_{\alpha}, W_{\alpha \beta}$ are defined over some finite extensions of the field $F$ which are also constructed by the algorithm). We (and the algorithm) represent every compound $W_{\alpha}$ or $W_{\alpha \beta}$ in two following manners: by its general point [16] and on the other hand by a certain system of algebraic equations such that the compound under consideration coincides with a variety of the roots of this system, in the similar case we say that the system determines the varie ty.

For functions $g_{1}, g_{2}, h_{1}, \ldots, h_{s}$ a relation $g_{1} \leqslant g_{2} P\left(h_{1}, \ldots, h_{s}\right)$ denotes further that $g_{1} \leqslant g_{2} P\left(h_{1}, \ldots, h_{s}\right)$ for an appropriate polsnomial $P$.

Let $W \subset \mathbb{P}^{n}(\bar{F})$ be a closed projective variety, $\operatorname{codim} \mathbb{p}^{n}(W)=m$, defined and irreducible over some field $F_{1}$ being a finite extension of $F$, denote by $F_{2}$ the maximal subfield of $F_{1}$ which is a separable extension of $F$. Let $t_{1}, \ldots, t_{n-m}$ be algebraically independent over $F$. A g e n e ral point of the variety $W$ can be given by the following fields isomorphism

$$
F\left(t_{1}, \ldots, t_{n-m}\right)[\theta] \sim F_{2}\left(X_{j_{1}} / X_{j_{0}}, \ldots, X_{j_{n-m}} / X_{j_{0}},\left(X_{0} / X_{j_{0}}\right)^{q^{q}}, \ldots,\left(X_{n} / X_{j_{0}}\right)^{q}\right) \subset F_{1}(W)(1)
$$


for suitable $q^{\nu}$ (here and further $\nu \geqslant 0$ when $q>0$ and we set $q^{\gamma}=1$ when $\operatorname{char}(F)=0$ ), index $0 \leqslant j_{0} \leqslant n$ and an element $\theta$ is algebraic separable over a field $F_{2}\left(t_{1}, \ldots, t_{n-m}\right)$; denote by $\phi(Z)$ its minimal polynomial such that $l_{Z}(\phi)=1$. The elements $X_{j} / X_{j_{0}}$ are considered herein as the rational functions on the varlety $W$, herewith $W$ is not situated in a hyperplane $\left\{X_{j_{0}}=0\right\}$, under the isomorphism (1) $t_{i} \rightarrow X_{j i} / X_{j_{0}}, 1 \leqslant i \leqslant n-m$. The algorithms further represent the isomorphism (1) by the images of rational functions $\left(X_{j} / X_{j_{0}}\right)^{q}$ in the field $F_{2}\left(t_{1}, \ldots, t_{n-m}\right)[\theta]$. Sometimes, when there is no misunderstanding, we identify a rational function with its image.

THBOREM 2. ( [2], [3], [4] ). a) An algorithm is suggested which for every compound $W_{\alpha}$ produces its general point and constructs a certain family of homogeneous polynomials $\psi_{1}^{(\alpha)}, \ldots, \psi_{N}^{(\alpha)} \in$ $\in F\left[X_{0}, \ldots, X_{n}\right]_{\text {such }}$ that a system $\psi_{1}^{(\alpha)}=\ldots=\psi_{N}^{(\alpha)}=0$ determines the variety $W_{\alpha}$. Denote $m=\operatorname{codim} W_{\alpha}, \theta_{\alpha}=\theta, \Phi_{\alpha}=\phi$. Then $q^{\gamma} \leqslant d^{2 m}, \operatorname{deg}_{z}\left(\phi_{\alpha}\right) \leqslant$

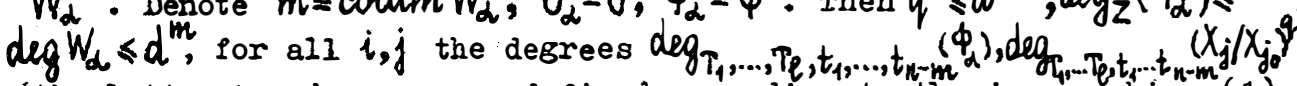
(the latter two degrees are defined according to the isomorphism (1) analogously to how $\operatorname{deg}_{T_{i}}(f)$ was defined above) are less than $d_{2} P\left(d^{m}, d_{1}\right)$, apart that $l\left(\phi_{\alpha}\right), l\left(\left(x_{j} / x_{j}\right)^{q^{\gamma}}\right) \leqslant\left(M_{1}+M_{2}+(n+l) \log d_{2}\right) P\left(d^{m}, d_{1}\right)$. A number of equations $N \leqslant m^{2} d^{4 m}$, the degrees $\operatorname{dog}_{x_{0}, \ldots, x_{n}}\left(\psi_{s}^{(\alpha)}\right) \leqslant d^{2 m}$

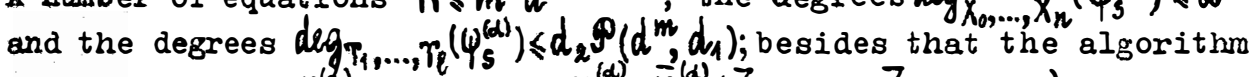
represents each $\psi_{s}^{(\alpha)}$ in a form $\psi_{s}^{(\alpha)}=\bar{\psi}_{s}^{(d)}\left(Z_{s, 0}, \ldots, Z_{s, n-m+2}\right)$ for suitable linear forms $Z_{s, j}$ in the variables $X_{0}, \ldots, X_{n}$ with the coeppiciente from $H$ and the pol jnomials $\bar{\psi}_{s}^{(\alpha)} \in F\left[Z_{s, 0}, \ldots, Z_{s, n-m+2}\right]_{s}$ thereto $\ell\left(\bar{\varphi}_{s}^{(d)}\right) \leqslant\left(M_{1}+M_{2}+(n+l) \log d_{2}\right) P\left(d^{m}, d_{1}\right)$, lastly the size, $L_{\ell}\left(Z_{b, j}\right) \leqslant \mathscr{P}\left(n, \log d d_{1} d_{\ell}\right)$ for all $s, j$. The total running time of the algorithm can be bounded from above by $\mathscr{P}\left(M_{1}, M_{2},\left(d_{1}^{n} d_{1} d_{2}\right)^{c+l}\right)$ Obviously, the latter value is less than $\mathcal{P}\left(L^{c+l}(q+1)\right) \leqslant \mathscr{P}\left(L^{\log ^{L} L}(q+1)\right)$ if $n=\theta(d)$.

b) An algorithm is suggested which for every absolutely irreducible compound $W_{\alpha \beta}$ finds the maximal separable subfield $F_{2}=F\left[\xi_{\alpha \beta}\right]$ of the minimal field of definition $F_{1}$ (containing $F$ ) of the variety $W_{\alpha \beta}$. The algorithm produces a general point of $W_{\alpha \beta}$ and some syatem of equations with the coefficients from the field $F_{2}$ determining the variety $W_{\alpha \beta}$. For the parameters of the general point and the system of equations hold the same bounds as in the item a) of the theorem. Denote by $\varphi_{\alpha \beta} \in F[Z]$ the minimal polynomial for $F_{\alpha \beta}$ such that $l_{c_{Z}}\left(\varphi_{\alpha \beta}\right)=1$, then $\operatorname{deg}_{z}\left(\varphi_{\alpha \beta}\right) \leqslant \operatorname{deg} W_{\alpha \beta}$ and the degrees $\operatorname{deg}_{T_{1}}, \ldots, T_{l}\left(\varphi_{\alpha \beta}\right) \leqslant d_{2} P\left(d^{m}, d_{1}\right)$, lastly $l\left(\varphi_{\alpha \beta}\right) \leqslant\left(M_{1}+M_{2}+\right.$ $\left.(n+l) \log d_{2}\right) P\left(d_{1}^{m} d_{1}\right)$. The time bound is the same as in the item a). 
REMARK. If we are supplied with a general point (with the same bounds on its parameters as in the theorem 2) of a closed irreducible variety $V_{1}=\pi\left(W_{d}\right)$ where $\pi\left(X_{0} \ldots . . X_{n}\right)=\left(X_{0}: \ldots: X_{m}\right)$ is a lenear projection $\pi: \mathbb{P}^{n} \rightarrow \mathbb{P}^{m}$ and $W_{\alpha}$ is some compound of the variety $\left\{f_{0}=\ldots=f_{k}=0\right\} \subset \mathbb{P}^{n}(F)$, then we can produce a system of equations determining $V_{1}$ with the same bounds on the parameters as for the family $\psi_{s}^{(\alpha)}$ in the theorem 2 within the same time bound.

In conclusion of the section 1. The authors make a conjecture that one can find the compounds wi.thin time $\mathcal{P}^{\prime}\left(d^{\left(c^{\prime}+l+1\right) n},\left(d_{1} d_{2}\right)^{n+l}, L\right)$ where $c^{\prime}=\max \min \left\{\operatorname{dim} W_{\alpha}+1, \operatorname{codim} W_{\alpha}\right\}$.

\section{Projecting a constructive set}

Let an input formula $\exists X_{1} \ldots \exists X_{S}\left(\& \&_{1 \leqslant j \leqslant k}\left(f_{j}=0\right) \&(g \neq 0)\right)$ be given, herein the parameters of the polynomials $f_{j}, g \in F\left[Z_{1}, \ldots\right.$, $\left.Z_{n-s}, X_{1}, \ldots, X_{s}\right]$ satisfy the same bounds as of $f_{j}$ in the section 1 . The goal in the present section is to produce on equivalent quantifierfree formula $V_{1 \leqslant i \leqslant N}\left(\&_{1 \leqslant j \leqslant x_{i}}\left(f_{i j}^{(1)}=0\right) \&\left(g_{i}^{(1)} \neq 0\right)\right) \quad$ where $f_{i j}^{(1)}, g_{i}^{(1)} \in F\left[Z_{1}, \ldots, Z_{n-s}\right]$.

The input formula is equivalent to $\exists X_{0} \exists X_{1} \ldots \exists X_{s} \exists X_{s+1}\left(\left(X_{0} \neq 0\right) \&\right.$ $\left.\&_{1 \leqslant j \leqslant k}\left(\bar{f}_{j}=0\right) \&\left(f_{0}=X_{s+1} \bar{g}-X_{0}^{1+\operatorname{deg} \bar{g}}=0\right)\right)$, therein $X_{0}, X_{S+1}$ are new variables and $f_{j}=X_{0} \operatorname{deg}_{X_{1}} X_{s}\left(f_{j}\right) f_{j}\left(Z_{1}, \ldots, Z_{n-s}, X_{1} / X_{0}, \ldots, X_{s} / X_{0}\right), \bar{g}=X_{0} \operatorname{deg}_{x_{1} \ldots x_{s}}(g)$ $g\left(Z_{1}, \ldots, Z_{n-s}, X_{1} / X_{0}, \ldots, X_{s} / X_{0}\right)(c 1$. [ [] ). The desired projection, i.e. the constructive set consisting of all the points $\left(z_{1}, \ldots, z_{n-s}\right) \in A^{n-s}(\bar{F})$ satisfying the latter formula, we denote by $\Pi$. One can assume further w.1.0.g. that $\operatorname{deg}_{x_{0}, \ldots, x_{s+1}} f_{j}=d-1,0_{x} \leqslant j \leqslant k$, replacing $f_{j}$ by the family of polynomials $\left\{f_{j} X_{i} d-1-d_{\text {eg }} f_{j}\right\}_{0 \leq i \leq s+1}$ Introduce a variety $U=\left\{\left(z_{1}, \ldots, z_{n-s} ;\left(x_{0} ; \ldots: x_{s+1}\right)\right) \in\left(A^{n-s} \times P^{s+1}\right)(\bar{F})\right.$; $\left.\&_{0 \leqslant j \leqslant K}\left(\bar{f}_{j}=0\right)\right\}$ and a natural linear projection $\pi: A^{n-s_{x}} \mathbb{P}^{s+1}$ $\rightarrow A^{n-s}$, then the desired $\Pi=\pi\left(U \cap\left\{X_{0} \neq 0\right\}\right)$. For each point $z=\left(z_{1}, \ldots, z_{n-s}\right) \in A^{n-s}(\bar{F})$ consider the variety(the layer) $U_{z}=\pi^{-1}(z) \cap U \subset\{z\} \times \mathbb{P}^{s+1} \simeq \mathbb{P}^{s+1}$. The condition $z \in \Pi$ is true iff for an appropriate $0 \leqslant m \leqslant s+1$ the layer $U_{Z}$ has at least one compound $W$ with the dimension $s+1-m$ such that $W \not \subset\left\{X_{0}=0\right\}$.

Fix a point $z$ in the followiag speculations for some time. It is not difficuit (see e.g. $\oint_{2}$ [2] ) to indicate a family of $N^{\prime}=$ $=k d^{m}+1$ vectors $u^{(1)}, \ldots, u^{\left(N^{\prime}\right)} \in H^{k+1}$ any $k+1$ from which are linearly independent (we suppose here and below that $H$ contains sufficiently many lement, extending it if necessary). Denote $h_{i}=$ $=\sum_{0 \leqslant j \leqslant k} u_{j}^{(i)} \bar{f}_{j}$, nerewith $u^{(i)}=\left(u_{0}^{(i)}, \ldots, u_{k}^{(i)}\right)$. The relevant compound $W$ of $U_{z}$ exists iff there are auch indices $1 \leqslant i_{1} \leqslant \ldots<i_{m} \leqslant N^{\prime}$ 
that $W$ is a compound of the variety $\left\{h_{i_{1}}(z)=\ldots=h_{i_{m}}(z)=0\right\} \subset \mathbb{P}^{3+1}$, herein the coordinates of the point $Z$ are substituted instead of $Z_{1}, \ldots, Z_{n-6}$, 1.e. $h_{i_{j}}(z) \in \bar{F}\left[X_{0}, \ldots, X_{s+1}\right]$ (cf. $\xi 4 a[2]$ ).

One can construct (see $\S 2$ [2] ) a family $\not Z=M_{s, s-m, d m}$ consisting of $(s-m+1)$-tuples of linear forms in variables $X_{1}, \ldots$, $X_{S+1}$ with the coefficients from $H$ such that for every variety $W_{1} \subset p^{s}$ satisfying the inequalities $\operatorname{dim} W_{1} \leqslant s-m, \operatorname{deg} W_{1} \leqslant d^{m}$ there is $(5-m+1)$-tuple $\left(Y_{1}, \ldots, Y_{5-m+1}\right) \in \mathcal{J l}$ for which $W_{1} \cap\left\{Y_{1}=\ldots\right.$ $\left.=Y_{s-m+1}=0\right\}=\varnothing_{\text {. Thereto }}$ card $(3 \gamma) \leqslant\left(\begin{array}{c}(s+1) d^{m}+1 \\ s-m\end{array}\right)$. Let us take a variety $W \cap\left\{X_{0}=0\right\}$ as $W_{1}$. Supplement Iinear forms $Y_{0}=X_{0}, Y_{1}$. $\ldots, Y_{s-m+1}$ up to a basis $Y_{0}, \ldots, Y_{s+1}$ with the coefficients from "H' of the space of linear forms in $X_{0}, \ldots, X_{5+1}$ (in arbitrary manner). Replacing variables denote $\hat{h}_{i}\left(z, Y_{0}, \ldots, Y_{s+1}\right)=h_{i}(z)$ and $\tilde{h}_{i}(z)=$ $=\hat{h}_{i}\left(z, Y_{0}, 0, \ldots, 0, Y_{s-m+2}, \ldots, Y_{s+1}\right)$. Thus, the condition under consideration about the existence of $W$ is equivalent to that there are indices $1 \leqslant i_{1}<\ldots<i_{m} \leqslant N^{\prime}$ and linear forans $Y_{1}, \ldots, Y_{s-m+1}$ for which the variety $\left\{\tilde{h}_{i_{1}}(z)=\ldots=\tilde{h}_{i_{m}}(z)=0\right\} \subset p^{m}$ as one of its compounds has a certain point $\widetilde{\Omega}=\left(\xi_{0}: \xi_{s-m+2}: \ldots: \xi_{s+1}\right)$ such that the point $\Omega=\left(z,\left(\xi_{0}: 0: \ldots: 0: \xi_{s-m+2}: \ldots: \xi_{s+1}\right)\right) \in U_{z} \cap\left\{Y_{0} \neq 0\right\}$ (in force of the theorem about the dimension of intersection [14]). Introduce a system of homogeneous algebraic equations

$$
\tilde{h}_{i_{j}}(z)-Y Y_{s-m+j+1}^{d-1}=0 ; \quad 1 \leqslant j \leqslant m
$$

in the rariables $Y_{0}, Y_{s-m+2}, \ldots, Y_{S+1}$ with the coefficients from $\vec{F}[Y] \subset \bar{F}(Y)=K \quad$ where $Y$ is algebraically independent over $F$. One can prove (see also lemma $11 \$ 5$ [3] ) that the set of roots in $\mathbb{P}^{m}(\bar{K})$ of the system(2) is finite. The variety of roots is decomposable on the irreducible and defined over $K$ nulldimensional compounds $V_{p_{K}}$ corresponding to the minimal prime ideals $p_{K} \subset K\left[Y_{0}, Y_{s-m+2}\right.$, $\left.\ldots, Y_{s+1}\right] /\left(\left\{\hbar_{i_{j}}(z)-Y Y_{s-m+j+1}\right\}_{1 \leqslant j \leqslant m}\right)$.The system (2) can be considered apart that as the system in the variables $Y, Y_{0}, Y_{s-m+2}, \ldots, Y_{s+1}$ with the coefficients from $F$ which determines a variety $\tilde{U}_{z}^{(F)} C$ $A^{m+l}(\tilde{F})$. It is not difficult to show (cf.lemma $12 \oint 5$ [3]) that there is a bijective correspondence between the points $V_{p_{k}}$ and on the other side such compounds $V_{P_{F}}$ of the variety $U_{z}^{(F)}$ that $V_{p_{F}}$ Is not contained in any union of finite number of hyperplanes of the kind $\left\{Y-c_{1}=0\right\} \subset A^{m+2}$ for $c_{1} \in \bar{F}$, notice that $\operatorname{dim} V_{p_{F}}=2$.

Non we exhibit an important audiliary device from [11] (see also $\oint_{3}[2]$ ). Let $g_{0}, \ldots, g_{k-1} \in F\left[X_{0}, \ldots, X_{n}\right]$ be homogeneous polynomials of degrees $\delta_{0} \geqslant \ldots \geqslant \delta_{k-1}$ reapectively. Introduce now variables 
$U_{0}, \ldots, U_{n}$ algebraically independent over $F\left(X_{0}, \ldots, X_{n}\right)$. Set $g_{k}=X_{0} u_{0}+\ldots+X_{n} U_{n} \in F\left(u_{0}, \ldots, U_{n}\right)\left[X_{0}, \ldots, X_{n}\right]$ and $D=\sum_{0 \leqslant i \leqslant n} \delta_{i}-n$, herein $\delta_{j}=1$ if $k \leqslant j \leqslant n$. Consider linear over $F\left(U_{0}, \ldots, U_{n}\right)$ mapping $O Q: B_{0} \oplus \ldots \oplus B_{K} \rightarrow B_{B}$ where $B_{i}$ (correspondingly $B_{\text {) }}$ is the space of homogeneous polynomials in $X_{0}, \ldots, X_{n}$ over the field $F\left(U_{0}, \ldots, U_{n}\right)$ of degree $D-\delta_{i} \quad$ (correspondingly $D$ ) for $0 \leqslant i \leqslant k$, namely $o l\left(b_{0}, \ldots, b_{k}\right)=\sum_{0 \leqslant i \leqslant k} b_{i} g_{i} . \quad$ Any element $b=\left(b_{0}, \ldots, b_{k}\right) \in$

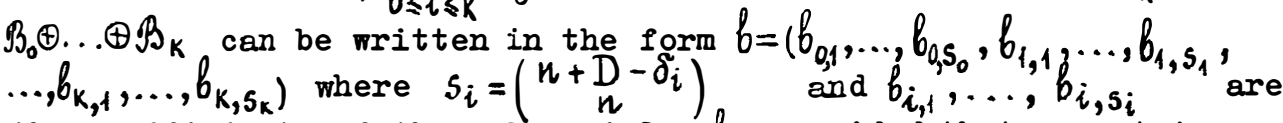

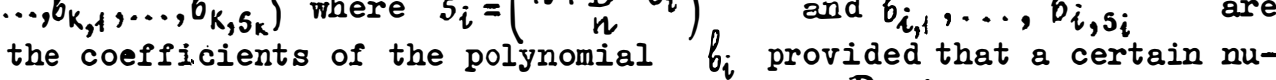
meration of all the monomials of the degree $D-\delta_{i}$ is fired. Analogously one can write the elements of the space $B$. In the chosen system of coordinates the mapping of has a matrice $A$ of the size $\left(\begin{array}{c}n+D \\ n\end{array}\right) \times\left(\sum_{0 \leqslant i \leqslant k} s_{i}\right)$. One can represent $A=\left(A^{\prime}, A^{\prime \prime}\right)$ where $A^{\prime}$ (call it the number part of $A$ ) contains $\sum_{0 \leqslant i \leqslant k-1} s_{i}$ columns and $A^{\prime \prime}$ (call it the formal part) contains $s_{k}$ columns, besides that the entries of $A^{\prime}$ belong to $F$, the entries of $A^{\prime \prime}$ are linear forms over $F$ in variables $U_{0}, \ldots, U_{n}$ (cf. [6]). There is proved in [10] that the system $g_{0}=\ldots=g_{k-1}=0$ has no roots in $\mathbb{P}^{n}(\bar{F})$ iff the ideal $\left(g_{0}, \ldots, g_{k-1}\right) \supset\left(X_{0}, \ldots, X_{n}\right)^{D}$. Besides that, the following proposition is ascertained in [11]. PROPOSITION: ( $[11]$ ). 1) The system $g_{0}=\ldots=g_{k-1}=0$ h has a finite number of roots in $\mathbb{P}^{n}\left(\bar{F}_{)}\right)$iff the rank $\operatorname{rg} A=\left(\begin{array}{c}n+D \\ n\end{array}\right)=r$; 2) all $r \times r$ minors of $A$ generate a principal ideal whose generator $R \in F\left[U_{0}, \ldots, U_{n}\right]$ is their g.c.d.;

3) the homogeneous form $R=\prod_{i \leqslant i \leqslant D_{1}} L_{i}$ (i) where $L_{i}=\sum_{0 \leqslant j \leqslant n} \xi_{j} U_{j}$ is a linear form over $\bar{F}$, moreover $\left(\xi_{0}^{(i)}: \ldots: \xi_{n}^{(i)}\right)$ is a root of the system and the number of occuring of the forms proportional to

$L_{i}$ for each $i$ in the product equals to the multiplicity of the corresponding root. Apart that $\operatorname{deg} R=D_{1}=r-\operatorname{rg}\left(A^{\prime}\right)$.

The algorithm designes the matrix $\AA$ with the entries from

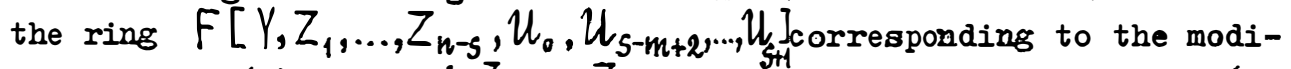
fied system (2) in which $Z_{1}, \ldots, Z_{k-5}$ are considered as variables (instead of $z_{1}, \ldots, z_{n-S}$ ) according to the just exhibited device. Denote by $A_{z}$ the matrix obtained from $A$ by means of substituting the coordinates of the point $Z$ instead of $Z_{1}, \ldots, Z_{n-s}$. Let the polynomial $R_{z} \in \bar{F}\left[Y, U_{0}, U_{s-m+2}, \ldots, U_{s+1}\right]$ correspond to the matrix $A_{z}$ as in the proposition. One can suppose w.1.0.8. that $Y \times R_{z}$ (dividing $R_{z}$ on the greatest possible power of the variable $Y$ ). 
Regard a certain representation of the union $U_{P_{F}} V_{p_{F}}=\left\{S_{0}=\ldots\right.$ $\left.=S_{k^{\prime}-1}=0\right\}$ for suitable polynomials $S_{i} \in \bar{F}\left[Y, Y_{0}, Y_{s-m+2}, \ldots, Y_{s+1}\right]$ homogeneous relatively to $Y_{0}, Y_{s-m+2}, \ldots, Y_{s+1}$. Considering a system $S_{i}\left(0, Y_{0}, Y_{s-m+2}, \ldots, Y_{s+1}\right)=0 ; 0 \leqslant i \leqslant K^{\prime}-1$ and basing on the proposition (see also lemma $16 \$ 5[3]$ ), one proves that $R_{z}\left(0, U_{0,} u_{s-m+2}, \ldots, u_{s+1}\right)=\prod_{i} L_{i}$ and moreover the linear forms $L_{i}=\Sigma_{j} \xi_{j}^{(i)} U_{j}$ correspond bijectively to the points $\left(\xi_{0}^{(i)}: \xi_{s-m+2}^{(i)}: \ldots: \xi_{s+1}^{(i)}\right) \in W_{z}^{\prime} \subset p^{m}$ where the cone $\operatorname{con}\left(W_{z}^{\prime}\right)=\left(U_{\gamma_{F}} V_{\gamma_{F}}\right) \cap\{Y=0\} \quad$. Thereupon it is not difficult to check that $\Omega \in W_{Z}^{\prime} \quad$ (cf. lemma 13 $\$ 5$ [3]). Summarizing and utilizing the notations introduced above, we have ascertained the following.

Larma 1. The formula $\exists X_{1} \ldots \exists X_{s}\left(\& \&_{1 \leqslant j \leqslant k}\left(f_{j}=0\right) \&(g \neq 0)\right)$ is valid in a point $z \in F^{n-s}$ iff for appropriate $0 \leqslant m \leqslant s+1$ there exist ouch indices $1 \leqslant i_{1}<\ldots<i_{m} \leqslant N^{\prime}$, a set of linear forms $\left(Y_{1}, \ldots, Y_{s-m+1}\right) \in M$ and a point $\Omega_{s}=\left(z,\left(\xi_{0}: 0: \ldots: 0: \xi_{s-m+2}: \ldots: \xi_{s+1}\right)\right)$ $\in U_{z} \cap\left\{X_{0} \neq 0\right\}$ (in the coordinates $Y_{0}, Y_{1}, \ldots, Y_{s+1}$ ) that the linear form $\left(\xi_{0} u_{0}+\xi_{s-m+2} u_{s-m+2}+\ldots+\xi_{s+1} u_{s+1}\right) \mid R_{z}\left(0, u_{0}, u_{s-m+2}, \ldots, u_{s+1}\right)$.

Non make more precise the definition of a version of Gaussian algorithm ( $V_{0} G_{0} a$ ) for reducing the matrices to the generalized trapezium form (cP. [7] ).V.G.a. is determined by a succession of pairs of indices (pivots) $\left(i_{0}, j_{0}\right),\left(i_{1}, j_{1}\right), \ldots,\left(i_{\rho}, j_{\rho}\right)$. Herewith $i_{\alpha} \neq i_{\beta}$ and $j_{\alpha} \neq j_{\beta}$ if $\alpha \neq \beta$ (o) Por any initial matrix $A^{(0)}$ v.Q.a. yields the chain of matrices $A^{(0)}, A^{(1)}, \ldots, A^{(j+1)}$. Introduce a notation $A^{(\alpha)}=\left(a_{i j}^{(\alpha)}\right)$. Apart that $a_{i_{\alpha j \alpha}}^{(\alpha)} \neq 0$ and $a_{i j}^{(\alpha+1)}=a_{i j}^{(\alpha)}+$

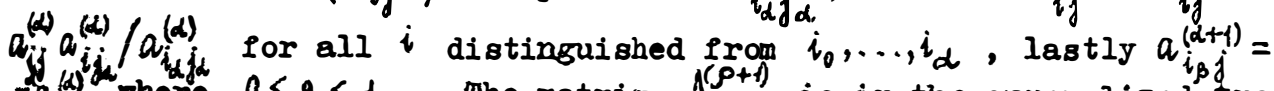
$=a_{i, j}^{(\alpha)}$ where $0 \leqslant \beta \leqslant \alpha$. The matrix $A^{(\rho+1)}$ is in the generalized trapezium form, namely, $a_{i j}^{(p+1)}=0$ when either $i$ differs from $i_{0}, \ldots, i_{\rho}$ or $i=i_{\alpha}, j=j_{\beta}$ and $\alpha>\beta$, besides that $a_{i_{\alpha} j_{\alpha}}^{(p+1)}=a_{i_{\alpha} j_{\alpha}}^{(\alpha)} \neq 0$. Denoto by $\Delta_{i j}^{(\alpha)}$ the deterrainant of $(\alpha+1) \times(\alpha+1)$ matrix formed by the rows with the indices $i_{0}, \ldots, i_{\alpha-1}, i$ and the columns with the Indices $j_{0}, \ldots, j_{\alpha-1}, j$ provided that $i \neq i_{0}, \ldots, i \neq i_{\alpha-1}$ and $j \neq j_{0}, \ldots, j \neq j_{\alpha-1}$. Then $a_{i j}^{(\alpha)}=\Delta_{i j}^{(\alpha)} / \Delta_{i_{\alpha-1} j_{\alpha-1}}^{(\alpha-1)} \quad$ (see e.g.
lemana 7 [7]). $A^{h-S^{2}}$ we turn ourselves to considering an arbitrary point $z \in$ $A^{h-s}$. Pix for some time $0 \leqslant m \leqslant s+1$ indices $1 \leqslant i_{1}<\ldots<i_{m} \leqslant N^{\prime}$ and a set of Iinear forms $\left(Y_{1}, \ldots, Y_{s-m+1}\right) \in M$ (se e Iemna 1). By

$v$ denote the number of rows of the matrix $A$. Produce a certain accesation of v.G.a.s $\Gamma_{1}, \Gamma_{2}, \ldots$ over a field $F\left(Y, Z_{1}, \ldots, Z_{n-5}, U_{0}\right.$, $\left.U_{s-m+2}, \ldots, U_{s+1}\right)$ and a auccession of polynomials $P_{1}, P_{2}, \ldots \in$ $F\left[Y, Z_{1}, \ldots, Z_{n-s}, U_{0}, U_{s-m+2}, \ldots, U_{s+r}\right]$ thereto v.G.a. $\Gamma_{i}$ can be applied 
correctly to the matrix $A_{z}$ for all points $z=\left(z_{1}, \ldots, z_{n-s}\right)$ of (possibly empty) quasiprojective variety ([14]) $W_{i} \subset A^{n-s}$ which is defined by the following conditions: inequality $0 \neq P_{i}\left(Y, z_{1}, \ldots\right.$, $\left.z_{n-s}, U_{0}, U_{s-m+2}, \ldots, U_{s+1}\right) \in F\left[Y, U_{0}, U_{s-m+2}, \ldots, U_{s+1}\right]$ and equalities $0=P_{j}\left(Y, z_{1}, \ldots, z_{n-s}, U_{0}, U_{s-m+2}, \ldots, U_{s+1}\right)$ for $1 \leqslant j \leqslant i-1$ are fulfilled. Apart that the variety $\left\{\left(z_{1}, \ldots, z_{n-s}\right): P_{i}\left(Y_{,} z_{1}, \ldots, z_{n-s}, U_{0}, U_{s-m+2}, \ldots, U_{s+1}\right)=0\right.$ for all $i\}=\varnothing, \quad$ henceforth $U_{i} W_{i}=A^{n-s}$. Exposed below construction is close to the proof of the lemma 9 [7].

Later on we apply the v.G.a.s $\Gamma_{1}, \Gamma_{2}, \ldots$ to the initial matrix $A$. As $\Gamma_{1}$ one can take an arbitrary v.G.a. Set a polynomial $P_{1}=\prod_{0 \leqslant \alpha \leqslant \rho_{1}}$ $\Delta i_{i}^{(d)} j_{d} \quad$ (for v.G.a. regarded at the current step the same notatiohs as above are utilized). Assume that $\Gamma_{1}, \ldots, \Gamma_{i} ; P_{1}, \ldots, P_{i}$ are already produced. Then as $\Gamma_{i+1}$ we take v.G.a. in which for every $0 \leqslant \alpha \leqslant \rho_{i+1}$ the column index $j_{\alpha}$ of the pivot in the matrix $A^{(\alpha)}$ is the least possible, moreover $j_{d}>j_{d-1}$ and the polynomials $P_{1, \ldots, P_{i}, \prod_{0 \leqslant \beta \leqslant \alpha}} \Delta_{i_{\beta} j_{\beta}}^{(\beta)}$ are linearly independent over $F$. FiV.G.a.s $\Gamma_{1}, \Gamma_{2}, \ldots$ whent it is 1 impossible to produce $\Gamma_{i+1}$ satisfying formulated above requirements (if $\rho_{i+1}<\tau-1$ then $W_{i+1}=\varnothing$ ).

One can ascertain that if $W_{i} \neq \varnothing$ then for each $z \in W_{i}$ the polynomial $R_{z}$ (see proposition) is obtained as the value in the point $z$ of the polynomial $\operatorname{det} \Delta_{i}$ (up to a factor $\gamma^{\varepsilon}$ for a suitable $\varepsilon$ ), where $y \times y$ submatrix $\Delta_{i}$ of the matrix $A$ is generated by the columns with the indices $j_{0}, \ldots, j_{q-1}$ corresponding to v.G.a. $\Gamma_{i}$ (d) This follows erom the fact that in the matrix $\left(A^{(\alpha)}\right)_{z}$ an entry $a_{\beta j}^{(\alpha)}=0$ when $\beta \neq i_{0}, \ldots, i_{\alpha-1}$ and $j<j_{\alpha}$ in force of the choice of $j_{d}$. Therefore, if for an appropriate $\alpha$ a cell $\left(i_{\alpha-1}, j_{\alpha-1}\right)$ belongs to the number part $A^{\prime}$ of $A$ and a cell $\left(i_{\alpha}, j_{\alpha}\right)$ belongs to the formal part $A^{\prime \prime}$ of $A_{p}$ then $\operatorname{rg}\left(\left(A^{\prime}\right)_{z}\right)=\alpha$ that implies the mentioned representation of $R_{z}$.

Write $\operatorname{det} \Delta_{i}=\Sigma_{\varepsilon} \Delta_{i}^{(\varepsilon)} \vee \varepsilon$, herewith $\Delta_{i}^{(\varepsilon)}\left(Z_{1}, \ldots, Z_{n-s}\right) \in F\left[Z_{1}, \ldots, Z_{n-s)}\right.$ $\left.U_{0}, U_{s-m+2}, \ldots, U_{s+1}\right]$. Introduce varieties $W_{i}^{(\varepsilon)}=\left\{\left(z_{1}, \ldots, z_{n-s}\right) \in W_{i}: \Delta_{i}^{(0)}\left(z_{1}\right.\right.$, $\left.\left.\ldots, z_{n-s}\right)=\ldots=\Delta_{i}^{(\varepsilon-1)}\left(z_{1}, \ldots, z_{n-s}\right)=0 ; \Delta_{i}^{(\varepsilon)}\left(z_{1}, \ldots, z_{n-s}\right) \neq 0\right\}$ for $\varepsilon \geqslant 0$. The variety $W_{i}^{(\varepsilon)}$ is quasiprojective as the intersection of two quasiprojective varieties, namely, if $\Xi^{(j)}=\left\{\&_{\beta}\left(G_{\beta}^{(j)}=0\right) \& V_{\gamma}\left(C_{\gamma}^{(j)} \neq 0\right)\right\} ; \quad j=1,2$ then $\left.\Xi_{1} \cap \Sigma_{2}=\left\{\& \beta_{\beta}^{(1)}, \beta^{(2)}\left(G_{\beta(1)}^{(1)}=0\right) \& G_{\beta}^{(2)}=0\right) \& V_{\gamma^{(1)}, \gamma^{(2)}}\left(C_{\gamma^{(1)}}^{(1)} C_{\gamma^{(2)}}^{(2)} \neq 0\right)\right\}$. Moreover $W_{i}^{\left(\varepsilon_{1}\right)} \cap W_{i}^{\left(\varepsilon_{2}\right)}=\varnothing$ for $\varepsilon_{1} \neq \varepsilon_{2}$ and $U_{\varepsilon} W_{i}^{(\varepsilon)}=W_{i}$. Thereupon represent $\Delta_{i}^{(\varepsilon)}=\sum_{0 \leqslant 1 \leqslant D_{2}} e_{i}^{(\varepsilon, j)} u_{0}^{D_{2}-j} \quad$ where $e_{i}^{(\varepsilon, j)}\left(Z_{1}, \ldots, Z_{n-s}\right) \in F\left[Z_{1}, \ldots, Z_{n-s}, U_{s-m+2}, \ldots, U_{s+1}\right]$. Consider quasiprojec- 
tive varieties $W_{i}^{(\varepsilon, j)}=\left\{\left(z_{4}, \ldots, z_{n-s}\right) \in W_{i}^{(\varepsilon)}: e_{i}^{(\varepsilon, x)}\left(z_{1}, \ldots, z_{n-s}\right)=0, \quad 0 \leqslant x<j ;\right.$ $\left.e_{i}^{(k, j)}\left(z_{1}, \ldots, z_{n-s}\right) \neq 0\right\}$, then $W_{i}^{\left(\varepsilon, j_{1}\right)} \cap W_{i}^{\left(\varepsilon, j_{2}\right)}=\varnothing$ when $j_{1} \neq j_{2}$ and $U_{0 \leqslant j \leqslant D_{2}} W_{i}^{(\varepsilon, j)}=$ $W_{i}^{(k)}$. Observe that the proposition and the ascertained earlier entail that $\left(\Delta_{i}^{(\varepsilon)}\right)_{z}=\Delta_{i}^{(\varepsilon)}\left(z_{1}, \ldots, z_{n-s}, U_{0}, U_{s-m+\ell}, \ldots, U_{s+1}\right)=\Pi_{x} L_{x}^{C_{x}} \quad$ is a product of linear forms for $z \in W_{i}^{(\varepsilon)}$. This implies that for $z \in W_{i}^{(\varepsilon, j)}$ the polynomial $\left(e_{i}^{(\varepsilon, j)}\right)_{z}$ equals to the product of powers $L_{x}^{C_{x}}$ of all linear forms $L_{x}$ in which the coefficient at $U_{0}$ vanishes. Henceforth $\left(e_{i}^{(\ell, i)}\right)_{z} \mid\left(\Delta_{i}^{(\varepsilon)}\right)_{z}$ in the ring $\bar{F}\left[U_{0}, U_{s-m+2}, \ldots, U_{s+1}\right]$. our nearest purpose is to calculate the quotient $\left(\Delta_{i}^{(\varepsilon)}\right)_{z} /\left(e_{i}^{(\varepsilon, j)}\right)_{z}$ for $z \in W_{i}^{(\ell, j)}$. If $I=\left(I_{s-m+2}, \ldots, I_{s+1}\right)$ is a multifindex then denote $U^{I}=U_{s-m+2}^{I_{s-m+2}} \ldots U_{s+1}^{I_{s+1}}$, apart that by $I<J$ denote the lexicographicaI order on multiindices. Write $e_{i}^{(\varepsilon, j)}=\Sigma_{I} \gamma_{I} U^{I}$ and let $0 \neq \gamma_{I} \in F\left[Z_{1}, \ldots, Z_{n-s}\right]$ for a certain $I$ (pixed in further speculations). Introduce a quasiprojective variety $W_{i, I}^{(\varepsilon, j)}=\left\{\left(z_{1}, \ldots, z_{n-s}\right) \in W_{i}^{(\varepsilon, j)}\right.$ :

$\gamma_{J}\left(z_{1}, \ldots, z_{n-s}\right)=0$ when $J>I$ and $\left.\gamma_{I}\left(z_{1}, \ldots, z_{n-s}\right) \neq 0\right\}$. Evidently $W_{i, I_{1}}^{(\varepsilon, j)} \cap W_{i, J_{1}}^{(\varepsilon, j)}=\varnothing \quad$ if $I_{1} \neq J_{1}$ and $U_{I_{1}} W_{i, I_{1}}^{(\varepsilon, j)}=W_{i}^{(\varepsilon, j)}$. For any point $\left(z_{1}, \ldots, z_{n-8}\right) \in W_{i, I}^{(\varepsilon, j)}$ the quotient $\left(\Delta_{i}^{(\varepsilon)}\right)_{z} /\left(e_{i}^{(\varepsilon, j)}\right)_{z}$ can be obtained by means of the described below process of dividing polynomial on polynomial and after that substituting the coordinates $Z_{1}, \ldots, Z_{n-s}$ instead of variables $Z_{1}, \ldots, Z_{n-s}$.

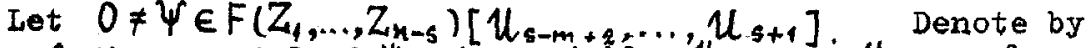
$l e x(\psi) \neq 0$ the monomial of $\psi$ in variabies $\|_{b-m+l}, \ldots, U_{\jmath+1}$ for which in $\psi-\operatorname{le} x(\psi)$ occur only the monomials less than $l e x(\psi)$, set $\bar{\psi}-\psi\left(u_{s-m+2}^{m}, U_{s-m+s}^{m-1}, \ldots, U_{s+1}\right)$ and $\sigma(\psi)=\operatorname{deg}(\bar{\psi})$. Delete from $e_{i}^{(\theta, j)}$ all the monomials $\gamma_{g} u^{J}$ (except $\gamma_{I} u^{I}$ ) with $\sigma\left(u^{J}\right) \geqslant \sigma\left(u^{I}\right)$ and denote obtained polynomial by $\widetilde{e}_{i}^{(\varepsilon, j)}$. Then $\left(e_{i}^{(\varepsilon, j)}\right)_{z}=\left(\widetilde{e}_{i}^{(\varepsilon, j)}\right)_{z}$, when $z \in W_{i, I}^{(\varepsilon, j)}$ since $\left(e_{i}^{(\varepsilon, j)}\right)_{z}$ is the product of linear forms. For any index $j<x \leqslant D_{2}$ the algorithm designs a succession of nonzero polynomials $\psi_{0}=e_{i}^{(\varepsilon, x)}, \psi_{1}, \ldots, \psi_{\rho}$. Represent uniquely $\psi_{t}=\psi_{t}^{(1)}+\psi_{t}^{(2)}+$

$+\psi_{t}^{(3)}$, herewith $\bar{\psi}_{t}^{(1)}, \bar{\psi}_{t}^{(2)}$ are homogeneous, $\sigma\left(\psi_{t}^{(3)}\right)<\sigma\left(\psi_{t}\right)=\sigma\left(\psi_{t}^{(1)}\right)=$ $-\sigma^{c}\left(\psi_{t}^{(t)}\right)$ and $\psi_{t}^{(1)} / u^{I} e F\left(z_{1}, \ldots, Z_{n-s}\right)\left[u_{s-m+2}, \ldots, u_{s+1}\right]$ each monomial from $\psi_{t}^{(t)}$ is not divided by $U^{I}$. Then $\psi_{t+1}=$ $=\gamma_{I}\left(\psi_{t}-\psi_{t}^{(2)}\right)-\psi_{t}^{(1)} \widetilde{e}_{i}^{(\varepsilon, j)} / U^{I}$ for every $0<t \leqslant \rho-1$ (obviously, $\sigma^{\prime}\left(\psi_{t+1}\right)<$ $\left.<r\left(\psi_{t}\right)\right)$. Regard a polymomial $\psi_{1, I}^{(\varepsilon, j, x)}=\sum_{0 \leq t \leq \rho-1} \psi_{t}^{(1)} \gamma_{I}^{\rho-t-1} / U^{I} \in$

$f\left[Z_{1}, \ldots, Z_{n-1}, U_{3-m+2, \ldots, j} U_{3+1}\right]$ and set $\psi_{i, I}^{(\varepsilon, j)=\gamma_{I}^{\rho}, t \leqslant \rho-1} u_{0}^{D_{2}-j}+\sum_{j<x \leqslant J_{2}} \psi_{i, I}^{(\varepsilon, j, x)} u_{0}^{D_{2}-x}$. one can check that $\left(e_{i}^{(\varepsilon, x)}\right)_{z} /\left(\gamma_{I}^{-\rho} e_{i}^{(\varepsilon, j)}\right)_{z}=\left(\psi_{i, I}^{(\varepsilon, j, x}\right)_{z}$ for $z \in W_{i, I}^{(\varepsilon, j)}$ and therefore $\left(\Delta_{i}^{(\varepsilon)_{z}} /\left(e_{i}^{(\varepsilon, j)_{z}}\right)_{z}=\left(\gamma_{I}^{-\rho} \psi_{i, I}^{(\varepsilon, j)}\right)_{z}\right.$ equals to the product of $L_{\mu}^{c_{\mu}}$ for all linear forms $L_{\mu}$ in which the coefficient 
at the variable $U_{0}$ does not vanish.

Thereupon remind that con $W_{Z}^{\prime}=U_{p_{F}} V_{\rho_{F}} \cap\{Y=0\} \quad$ and int-

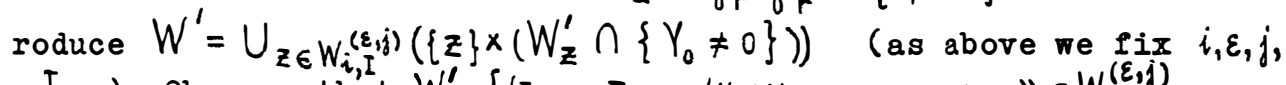

$I)$. Observe that $W^{\prime}=\left\{\left(z_{1}, \ldots, z_{n-s},\left(y_{0} ; y_{s-m+2}: \ldots: y_{s+1}\right)\right) \in W_{i, I}^{(\varepsilon, j)} \times\right.$

$A^{m}(\bar{F}) \subset W_{i, I}^{(\varepsilon, j)} \times \mathbb{P}^{m}(\bar{F}): 0=\left(\varphi_{i, I}^{(\varepsilon, j)}\left(-\Sigma_{s-m+2 \leqslant \alpha \leqslant s+1} u_{\alpha} y_{\alpha}, y_{0} u_{s-m+2}, \ldots, y_{0} u_{s+1}\right)\right)_{\varepsilon}$

$\left.\in \bar{F}\left[u_{s-m+2}, \ldots, u_{s+1}\right]\right\}$. Representing the polynomial

$\psi_{i, I}^{(\varepsilon, j)}\left(-\sum_{s-m+2 \leqslant d \leqslant s+1} U_{\alpha} Y_{\alpha}, Y_{0} U_{s-m+2}, \ldots, Y_{0} U_{s+1}\right)=\Sigma_{J} E_{J} U^{J} \quad$ leads to

an equality $W^{\prime}=\left\{\&_{J}\left(E_{J}=0\right)\right\} \cap\left(W_{i, i}^{(\varepsilon, j)} \times A^{m}\right)$. Because of $W^{(\varepsilon, j)}$ that

the subset $W^{\prime}$ is closed in the quasiprojective variety $W_{i, I}^{(\varepsilon, j)} \times \mathbb{A}_{i n-s}^{m}$

Consider the natural linear projection $\pi_{2}: A^{n-s} \times\left(\mathbb{P}^{m} \cap\left\{Y_{0} \neq 0\right\}\right) \rightarrow A^{n-s}$

defined by the formula $g_{2}\left(Z_{1}, \ldots, Z_{n-s},\left(Y_{0}: Y_{s-m+2}: \ldots: Y_{s+1}\right)\right)=\left(Z_{1}, \ldots, Z_{n-s}\right)$.

Let a morphism $\pi_{1}: W^{\prime} \rightarrow W_{i, I}^{(\varepsilon, j)}$ be the restriction of $\pi_{2}$ on $W^{\prime}$ !

our nearest goal is to show that $g_{1}$ is finite ( [14] ). Obviously, the inverse image $\pi_{1}^{-1}(V) \subset W^{\prime}$ of any open affine subset $V \subset W_{i, I}^{(\varepsilon, j)}$ is isomorphic to $\left(V \times \mathbb{A}^{m}\right) \cap W^{\prime}$, henceforth $\pi_{1}^{-1}(V)$ is open in

$W^{\prime}$ and besides that $\pi_{1}^{-1}(V)$ is affine since $\pi_{1}^{-1}(V)$ is closed in the open affine set $\bigvee \times A^{m} \quad([14])$. Now we check that every coordinate function $Y_{\mathfrak{X}} / \gamma_{0}$ on the variety $\pi_{1}^{-1}\left(V_{)}\right.$sqtisfies a suitable relation of integral dependence over the ring $\bar{F}[V]$ where $s-m+2 \leqslant x \leqslant s+1$. Let $\psi_{i, I}^{(\varepsilon, j)}=\psi_{i, I}^{(\varepsilon, j)}\left(U_{0}, U_{c m+2}, \ldots, U_{s+1}\right)$. Then $\psi_{i, I}^{(\varepsilon, j)}\left(Y_{x} / Y_{0}, 0, \ldots, 0,-1,0, \ldots, 0\right)=0$ on $W^{\prime}$, herein -1 is substituted instead of the variable $U_{\Re}$. Taking into account that $\left(\gamma_{I}\right)_{z} \neq 0$ when $z \in W_{i, I}^{(\varepsilon, j)}$ this yields an equation of integral dependence. So, we infer that the morphism $\delta_{1}$ is finite.

Utilizing the notations from the lemma 1 one concludes that a set $V_{i, I}^{(\varepsilon, \gamma)}$ consisting of all such points $Z=\left(z_{1}, \ldots, z_{n-s}\right) \in W_{i, I}^{(\varepsilon, j)}$ that there exists , point $\Omega=\left(z,\left(\xi_{0}: 0: \ldots: 0: \xi_{s-m+2}: \ldots: \xi_{s+1}\right)\right) \in U_{z} \cap\left\{X_{0} \neq 0\right\}$ is closed in $W_{i, I}^{(\varepsilon, j)}$ as $V_{i, I}^{(\varepsilon, j)}$ coincides with the image under projection $\pi_{l_{1}}$ of the closed in the domain of definition of $\pi_{l_{1}}$ (i.e. in $\left.W^{\prime}\right)$ set $\pi_{1}^{-1}\left(W_{i, I}^{(\varepsilon, j)}\right) \cap\left\{\tilde{f}_{0}=\ldots=\tilde{f}_{k}=0\right\}$ where $\tilde{f}_{x}\left(Y_{0}, Y_{5-m+l}\right.$, $\left.\ldots, Y_{s+1}\right)=\hat{f}_{x}\left(Y_{0}, 0, \ldots, 0, Y_{s-m+2}, \ldots, Y_{s+1}\right)$ and $\hat{f}_{x}\left(Y_{0}, Y_{1}, \ldots, Y_{s+1}\right)=\bar{f}\left(Z_{1,}, \ldots, Z_{n-s}, X_{0}, \ldots, X_{s+1}\right)$ for $0 \leqslant x \leqslant K$ and since the image of the closed set under a finite morphism is again closed ( [14]).

Now we describe a procedure $f_{(\mathcal{k}, j)}$ constructing the required $V_{i, I}^{(\xi, j)}$. Let the quasiprojective variety $W_{i, I}^{(\varepsilon, j)}=\left\{\&_{\beta}\left(G_{\beta}=0\right) \&\left(V_{\gamma}\left(C_{\gamma} \neq 0\right)\right)\right\}$, herewith the polynomials $G_{\beta}, C_{\gamma} \in F\left[Z_{1}, \ldots, Z_{n-s}\right]^{i, I}$ were actually produced 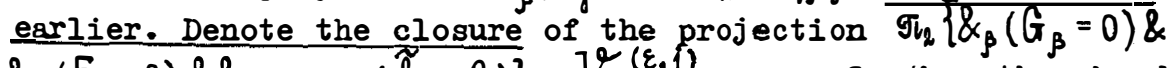 $\left.\& g\left(E_{j}=0\right) \& \& 0 \leqslant x \leqslant k\left(f_{x}=0\right)\right\}=V_{i, I}(\varepsilon, f) \quad$ On the other hand in force of the aforesaid the equalities hold $V_{i, I}^{\left(\varepsilon_{1}, j\right)}=V_{i I}^{(\varepsilon, j)} \backslash\left\{\& \gamma\left(C_{\gamma}=0\right)\right\}$ 

$=\bigvee_{i, I}^{(\varepsilon, j)} \backslash\left\{\&_{\gamma}\left(C_{\gamma}=0\right)\right\} \quad$. Thus, it remains only to design the af-
Iine rariety $\bigvee_{i, I}(\varepsilon, j)$

Involving the theorem 2 (see section 1) the algorithm finds the general points of the compounds $F$ of the variety $\left\{\&_{\beta}\left(G_{\beta}=0\right) \& \&_{J}\left(E_{J}=0\right)\right.$

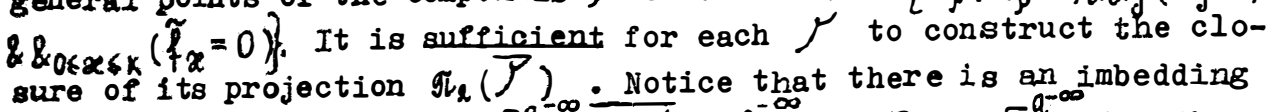
of the pields of punctions $F q^{-\infty}\left(\overline{\left.\pi_{2}(\mathcal{F})\right)}\right)=F^{-\infty}\left(Z_{1}, \ldots, Z_{n-s}\right) \in F^{q^{-\infty}}\left(Z_{1}, \ldots, Z_{n-s}\right.$, $\left.Y_{1} / Y_{0}, \ldots, Y_{s+1} / Y_{0}\right)=F^{-\infty}\left(\mathcal{q}^{\mu}\right)$. Therefore, the algorithm can produce the general point of $\overline{g_{2}(\mu)}$ yielding firstly a trascendental basis and after that a primitive element (cf.(1), section 1). Searching a transcendental basis and also a primitive element is based on the procedure for calculating a polynomial relation over $F_{\infty}$ (if it exists) between the elements $a_{1}, \ldots, a_{\rho+1} \in F\left(t_{1}, \ldots, t_{n-m_{1}}\right)[\theta] \subset F^{-\infty}(J)$ provided that $a_{1}, \ldots, a_{\rho}$ are algebraically independent over $F$, the procedure in its turn is reducible to solving a linear system whose indeterminates are the coefficients of the relation (cf. $\$ 1$ [2],

$\$ \$ 4 b, 6[3])$. Thereupon with the help of the remark just after the theorem 2 the algorithm computes a representation $\overline{\pi_{q}(\Gamma)}=\left\{\&_{g}\right.$ $\left.\left(B_{j}=0\right)\right\}$ where the polynomials $B_{g} \in F\left[Z_{1}, \ldots, Z_{n-s}\right]$.

Te summarize the results of the present section in the follow1 as lems, in which bounds are obtained making use of the theorem 2.

IRMMA 2. An algorithm is suggested which outputs the construct1 ve set $\Pi=g\left(U n\left\{X_{0} \neq 0\right\}\right)=\left\{\left(z_{1}, \ldots, z_{n-s}\right) \in A^{n-s}(\bar{F}): \exists X_{1} \ldots \exists X_{s}\left(\&_{1 \leqslant x \leqslant k}\left(f_{x}\left(z_{1}, \ldots, z_{n-s}\right.\right.\right.\right.$,

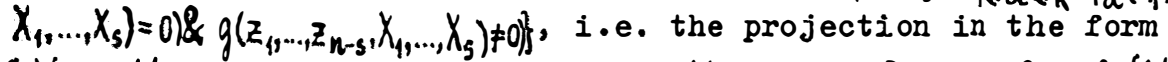

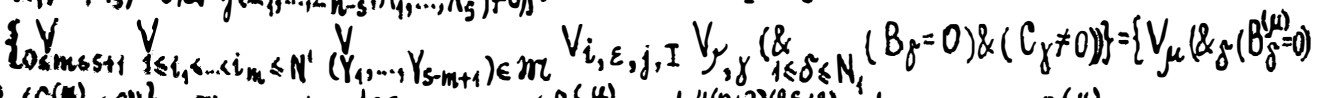
\& $\left.\left(C^{(\mu)} \neq(0)\right)\right\}$. Thereat $\operatorname{deg}_{Z_{1}, \ldots, Z_{n-s}}\left(B_{\delta}^{(\mu)}\right) \leqslant d^{4(n+2)(2 s+3)}, \operatorname{deg}_{T_{1}, \ldots, T_{l}}\left(B_{\delta}^{(\mu)}\right) \leqslant$ $d_{2} P\left(d^{(s+1) n}, d_{1}\right)$, lengths of descriptions $l(B(\mu)) \leqslant\left(M_{1}+M_{2}+(n+l) \log d_{2}\right) x$ $P\left(d^{(s+1) n}, d_{1}\right)$. Apart that $\operatorname{deg}_{z_{1}, \ldots, z_{n-s}}\left(C^{(\mu)}\right) \leqslant(3 d)^{(2 s+3)}, \operatorname{deg}_{T_{1}, \ldots, T_{l}}\left(c^{(\mu)}\right) \leqslant$ $d_{2} \rho\left(d^{(s+1)}, d_{1}\right)$ and $l\left(c^{(\mu)}\right) \leqslant\left(M_{1}+M_{2}+(n+l) \log d_{2}\right) \rho\left(d^{(s+1)}, d_{1}\right)$. Besides that, $\delta^{2}<(s+1)^{2}(3 d)^{4(2 s+3)(n+2)}, \mu \leqslant d^{12(s+2)(n+s+3)}$. The running time of the algorithm can be estimated by $P\left(M_{1}+M_{2}, d^{\operatorname{sn}(n+l)},\left(d_{1}+d_{2}\right)^{n+l}, q\right)$.

3. Subexponential-time deciding the first order theory of algebraically closed fields

Let a Boolean formula $Q$ with $N$ atoms of the kind $f_{i}=0$ where $f_{i} \in F\left[X_{1}, \ldots, X_{n}\right]$ satisfies the same bounds as in the section 1 , be given, $L_{l}(Q)$ denotes the size of $Q$. Firstly we exhibit a procedure reducing $Q$ to a disjunctive normal form. 
Following [7] name $\left(g_{1}, \ldots, g_{\rho}\right)$-cell for $g_{1}, \ldots, g_{\rho} \in F\left[X_{1}, \ldots, X_{n}\right]$ any nonempty quasiprojective variety of the kind $\left\{\varepsilon_{j} \in y_{1}\left(g_{j}=0\right) \&\right.$ $\left.\&_{j \in y_{2}}\left(g_{j} \neq 0\right)\right\} \subset \mathbb{A}^{n}(\bar{F})$, herewith $y_{1} \cup y_{2}=\{1, \ldots, \rho\}, y_{1} \cap y_{2}=\varnothing$. By means of the Bezout inequality [14] it is ascertained in [7] that a number of all $\left(g_{1}, \ldots, g_{\rho}\right)-$ cells is less or equal to $\left(1+\operatorname{deg} g_{1}+\ldots\right.$ $\left.+\operatorname{deg} g_{\rho}\right)^{n}$. We shall describe the method for decomposing the space $A^{n}$ on $\left(g_{1}, \ldots, g_{\rho}\right)$-cells by recursion on $\rho$. Assume that we are supplied with all $\left(g_{1}, \ldots, g_{\rho-1}\right)$-cells $(\rho \geqslant 1)$. Every $\left(g_{1}, \ldots, g_{\rho}\right)$-cell is of the form either $K \cap\left\{g_{\rho}=0\right\}$ or $K \cap\left\{g_{\rho} \neq 0\right\}$ for a pertinent $\left(g_{1}, \ldots, g_{\rho-1}\right)$-cell $K$. Henceforth it is sufficient to pick out (involving the theorem 2 from the section 1) all nonempty sets among quasiprojective varieties of the forms $K \cap\left\{g_{\rho}=0\right\}$ and $K \cap\left\{g_{\rho} \neq 0\right\}$. Applying the just described method the algorithm yields all $\left(\left\{f_{i}\right\}_{1 \leqslant i \leqslant N}\right)$-cells. Again repeatedly making use of the theorem 2 by induction on the number of logical signs in $Q$ the algorithm for each $\left(\left\{f_{i}\right\}_{1 \leqslant i \leqslant N}\right)$-cell checks, whether this call is contained in the constructive set $\Pi_{Q}=\{Q\} \subset \mathbb{A}^{n} \quad$ deternined by the formula $Q$, and thereby represents $\Pi_{Q}$ as a union of $\left(\left\{f_{i}\right\}_{1 \leqslant i \leqslant N}\right)$-cells $K^{(\mu)}$ that means reducing $Q$ to a disjunctive normal form $V_{\mu}(\& \delta \geqslant 1$ $\left.\left.\left(f_{\delta}^{(\mu)}=0\right) \&\left(f_{0}^{(\mu)} \neq 0\right)\right)\right)$. Moreover $1 \leqslant \mu \leqslant(1+N d)^{n}, 1 \leqslant \delta \leqslant N$, any polynomial $f_{f}(\mu)=f_{i}$ for a relevant $i$ and $f_{0}(\mu)=\prod_{j \in y} f_{j}$ for an appropriate $y \subset\{1, \ldots, N\}$. The working time of the exhibited procedure can be estimated according to the theorem 2 by $P\left(L_{2}(Q), N^{n},\left(d^{n} d_{1} d_{2}\right)^{n+l}, q\right)$.

Finally we pass to the general case. Let an input formula of the first order theory

$$
\exists Z_{1,1} \ldots \exists Z_{1, s_{1}} \forall Z_{2,1} \ldots \forall Z_{2, s_{2} \ldots} \ldots Z_{a, 1} \ldots \exists Z_{a, s_{Q}} Q
$$

be given where the formula $Q$ is of the kind as at the beginning of the section, $f_{i} \in F\left[Z_{1}, \ldots, Z_{s_{0}}, Z_{1,1}, \ldots, Z_{a, s_{a}}\right]$, herein $Z_{1}, \ldots, Z_{s_{0}}$ occur free, $n=s_{0}+s_{1}+\ldots+s_{a}$, by $L_{l}$ denote the size of (3). Applying to (3) alternatively the just exhibited procedure for reducing to a disjunctive normal form and the lemma 2 (section 2) the algorithm arrives after performing $x$ steps at en equivalent to (3) formula

$$
\exists Z_{1,1} \ldots \exists Z_{1, s_{1}} 7 \ldots \exists Z_{a-x, 1} \ldots \exists Z_{a-x, s_{a-x}} T\left(V_{1 \leqslant i \leqslant N}(x)\left(\&_{1 \leqslant j \leqslant k^{(x)}-1}\left(f_{i j}^{(x)}=0\right) \&\left(f_{i 0}^{(x)} \neq 0\right)\right)\right) .
$$

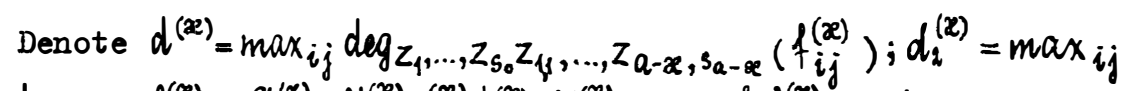
$\operatorname{deg}_{T_{1}, \ldots, T_{l}}\left(f_{i j}^{(x)}\right) ; g(x)=N^{(x)} k^{(x)} d^{(x)} ; M_{2}^{(x)}=\max _{i j} l\left(f_{i j}^{(x)}\right) ; \sigma=s_{a-x+1}$. Then in force of the theorem 2 and the lemma 2 the inequalities hold: $d^{(x)} \leqslant$ 


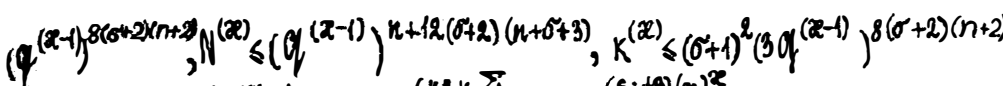
of $^{(x)} \leqslant\left(q^{(x-1)}\right)^{48 n(\sigma+8)} \leqslant(N d)^{\left(48 n \sum_{a-\alpha)+1} \leq j \leqslant a^{\left.\left(s_{j}+\theta\right) / x\right)^{x}}\right.}$. Apart that $d_{2}^{(x)} \leqslant d_{2}^{(x-1)} x$ $x \rho\left(q_{1}^{(x-1)}, d_{1}\right) \leqslant d_{2} \rho\left(q^{(x)}, d_{1}^{(x)}\right), M_{2}^{(x)} \leqslant\left(M_{1}+M_{2}+l \log d_{2}\right) \rho\left(q_{1}^{(x)}, d_{1}^{x}\right)$. Lastly the runoing time of the algorithm (after $\mathscr{W}$ steps) is less than

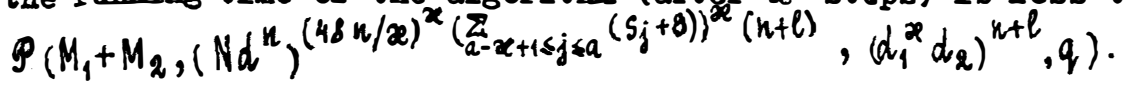

Performing $a$ steps completes the proof of the following THEOREY 3. An algorithr is proposed which for a formula (3) outputs an equivalent to it a quantifier-free one $V_{1 \leqslant i \leqslant N}\left(\&_{1 \leqslant j \leqslant K}\right.$ $\left.\left(g_{i j}=0\right) \&\left(g_{i 0} \neq 0\right)\right)$ where $g_{i j} \in F\left[Z_{1}, \ldots, Z_{s_{0}}\right]$, herewith $\operatorname{deg} z_{1}, \ldots, z_{s_{0}}\left(g_{i j}\right)$ $\leqslant\left(N d^{n}\right)^{(48 n(n+8 a) / a)^{a}}=D, \operatorname{deg}_{T_{1}, \ldots, T_{l}}\left(g_{i j}\right) \leq d_{2} 9\left(D, d_{1}^{a}\right) ; \quad$ besides that $\ell\left(g_{i j}\right) \leqslant\left(M_{1}+M_{2}+l \log d_{2}\right) \rho\left(D, d_{1}^{a}\right)$ - The integers $\mathcal{N}, \mathcal{K} \leqslant D$. Pinally, the algorithm works within the time $\rho\left(L_{2}, L_{2}(\varphi),\left(N d^{n}\right)(48 n(n+8 a) / a)^{a}(n+l)\right.$, $\left.\left(d_{1}^{a} d_{2}\right)^{n+l}, q\right)$.

REPERENCES

1. Chistor A.I., Grigor'er D.Yu. Polynomial-time factoring of the multivariable polynomials over a global field. - LOMI preprint B-5-82, Leningrad, 1982.

2. Chistor A.I., Grigor'er D.Yu. Subexponential-time solving systems of algebraic equations. I. - LOMI preprint E-9-83, Leningrad, 1983.

3. Chistor A.I., Grigor'er D.Yu. Subexponential-time solving systems of algebratc equations. II. - LOMI preprint E-10-83, Leningrad, 1983.

4. Chistor A.I., Grigor'er D.Yu. Polynomial-time factoring of polynomials and subexponential-time solving systems and quantifier elimination. - Notes of Scientific seminars of IOMI, Leningrad, 1984, vol.137.

5. Collins G.B. Quantifier elimination for real closed fields by cylindrical algebraic decomposition. - Lect.Notes Comput.Sci., 1975, rol.33, p.134-183.

6. Grigor'ev D.Yu. Multiplicative complexity of a bilinear form over a commutative ring. - Lect.Notes Comp.Sci., 1981, vol.118, p.281286.

7. Haintz J. Definability and fast quantifier elimination in algebraically closed fields. - Prepr.Univ.Prankfurt, Weat Germany, December, 1981. 
8. Kaltofen E. A polynomial reduction from multivariate to bivariate integral polynomial factorization. - Proc.14-th ACM Symp.Th. Comput., May, N.Y., 1982, p.261-266.

9. Kalt ofen E. A polynomial-time reduction from bivariate to univariate integral polynomial factorization. - Proc.23-rd IEEE Symp.Found Comp.Sci., October, N.Y., 1982, p.57-64.

10. Lazard D. Algébre linéaire sur $k\left[X_{1}, \ldots, X_{n}\right]$ et élimination. Bull.Soc.Math.France, 1977, vol.105, p.165-190.

11. Lazard D. Résolutions des systèmes d'équations algébriques. Theor Comput.sci., 1981, vol.15, p.77-110.

12. Lenstra A.K., Lenstra H.W., Lovasz L. Factoring polynomials with rational coefficients. - Math.Ann., 1982, vol.261, p.515-534.

13. Lenstra A.K. Factoring multivariate polynomials over finite fields. - Preprint Math.Centrum Amsterdam, IW 221/83, Februari, 1983.

14. Shafarevich I.R. Basic algebraic geometry. - Springer-Verlag, 1974.

15. Wüthrich H.R. Ein Entscheidungsverfahren für die Theorie der reellabgeschlossenen Körper. - Lect.Notes Comput.Sci., 1976, vol.43, p.138-162.

16. Zariski 0., Samuel P. Commutative algebra, vol.1, 2. - van Nostrand, 1960. 